\title{
Continuous erector spinae plane block versus intercostal nerve block in patients undergoing video-assisted thoracoscopic surgery: a pilot randomized controlled trial
}

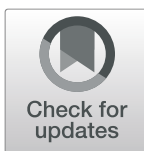

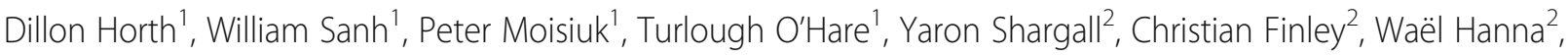
John Agzarian², Mauricio Forero ${ }^{1}$, Kim Davis ${ }^{3}$, Thuva Vanniyasingam', Lehana Thabane ${ }^{4}$ and Harsha Shanthanna ${ }^{*^{*}}$ (D)

\begin{abstract}
Background: The optimal analgesia method in video-assisted thoracoscopic surgery (VATS) remains controversial. Intercostal nerve blockade (ICNB) is limited by its duration of action. The erector spinae plane (ESP) block has the potential to provide satisfactory analgesia for VATS; however, the effectiveness of continuous ESP versus surgeonperformed ICNB has not been investigated. The objectives of this study were to establish feasibility of patient recruitment and follow-up before undertaking a fully powered randomized controlled trial (RCT); and, secondarily, to compare opioid usage, pain control, and sensory blockade.

Methods: This feasibility RCT was undertaken at St Joseph's Hospital, Hamilton, Ontario, Canada, and included 24 patients (>18 years) having elective VATS with at least one overnight stay. Exclusion criteria were patient refusal, body mass index $>40 \mathrm{~kg} / \mathrm{m}^{2}$, contraindications to neuraxial analgesia techniques as per the American Society of Regional Anesthesia and Pain guidelines, known allergy to local anesthetics, language or comprehension barriers, procedures with a higher chance of open surgery, and regular opioid use for $\geq 3$ months preoperatively. Patients underwent either continuous ESP ( $n=12)$ or surgeon-performed ICNB $(n=12)$. All patients received routine intraoperative anesthesia care and multimodal analgesia. Feasibility criteria were recruitment rate of two patients/ week and full follow-up in all patients in-hospital. We compared opioid consumption, postoperative pain scores (010 numerical rating scale), adverse events, patient satisfaction, and distribution of sensory blockade as clinical outcomes (secondary).

Results: Feasibility of primary outcomes was successfully demonstrated. Five patients had an epidural in anticipation of open surgery. Mean opioid consumption as equivalent morphine units was less in the ESP group over the first $24 \mathrm{~h}$ (mean difference, 1.63 [95\% Cl -1.20 to 4.45]) and $48 \mathrm{~h}$ (mean difference, 2.34 [95\% $\mathrm{Cl}-1.93$ to 6.61]). There were no differences in adverse effects.

(Continued on next page)
\end{abstract}

\footnotetext{
* Correspondence: shanthh@mcmaster.ca

'Department of Anesthesia, McMaster University, Hamilton, Ontario, Canada

Full list of author information is available at the end of the article
}

\section{$\triangle B M C$}

(c) The Author(s). 2021 Open Access This article is licensed under a Creative Commons Attribution 4.0 International License, which permits use, sharing, adaptation, distribution and reproduction in any medium or format, as long as you give appropriate credit to the original author(s) and the source, provide a link to the Creative Commons licence, and indicate if changes were made. The images or other third party material in this article are included in the article's Creative Commons licence, unless indicated otherwise in a credit line to the material. If material is not included in the article's Creative Commons licence and your intended use is not permitted by statutory regulation or exceeds the permitted use, you will need to obtain permission directly from the copyright holder. To view a copy of this licence, visit http://creativecommons.org/licenses/by/4.0/ The Creative Commons Public Domain Dedication waiver (http://creativecommons.org/publicdomain/zero/1.0/) applies to the data made available in this article, unless otherwise stated in a credit line to the data. 
(Continued from previous page)

Conclusions: A fully powered RCT is feasible with modifications. Our results also suggest that continuous ESP is safe and can decrease opioid needs. However, it is important to consider procedures to improve compliance to protocol and adherence to assigned interventions.

Trial registration: Clinicaltrials.gov identifier: NCT03176667. Registered June 5, 2017.

Keywords: Erector spinae plane block, Intercostal nerve blockade, Perioperative analgesia, Video-assisted thoracoscopic surgery

\section{Introduction}

Video-assisted thoracoscopic surgery (VATS) is an increasingly popular technique in thoracic surgery with improvements in technology. VATS provides significant advantages over open thoracotomy procedures including reduced surgical pain, reduced mortality, improved postoperative pulmonary function, and shorter hospital length of stay [1-4]. Nevertheless, there is still considerable amount of postoperative acute pain with VATS lobectomies. Controlling postoperative pain is crucial because increased acute pain has been associated with the development of chronic pain [5], increased hospital length of stay [6], and decreased patient satisfaction [7].

Most patients receive thoracic epidural analgesia (TEA) to treat postoperative pain in thoracotomy procedures [8]. For VATS procedures, the merits of these regional techniques are uncertain as compared with patient-controlled analgesia (PCA) with opioids [9-11]. The paravertebral block (PVB) is an alternative to TEA; however, both TEA and PVB have the potential for serious side effects and complications including severe hypotension, epidural bleeding, and spinal cord or nerve injury. Although PVB is associated with a lower incidence of hypotension, it carries the risk of pneumothorax, pleural and vascular puncture, and higher systemic absorption of local anesthetics. PVB is also technically challenging and does not always reliably spread in the paravertebral space [12]. In addition, placement of both TEA and PVB requires appropriate discontinuation of anticoagulants. Intercostal nerve blocks (ICNBs) have been used as an analgesic alternative to TEA and PVB in VATS with beneficial effect $[13,14]$. However, although ICNBs have shown to lower pain scores in the early postoperative period [15], they have a limited duration of effect [16]. A novel regional technique, the erector spinae plane (ESP) block, has been recently described [17]. This interfascial block involves ultrasound (US)-guided injection of local anesthetics posterior to the erector spinae muscle and superficial to the transverse process of thoracic vertebrae at the appropriate level. The ESP block predominantly targets the dorsal rami of the spinal nerves as they leave the intervertebral foramen, but also has the potential to block ventral rami [18]. Cadaveric examination of ESP block showed extensive cranial-caudal spread of the block, approximately four dermatomes above and below the site of injection. The successful use of the ESP block has led to studies in multiple clinical settings including abdominal surgery [17], VATS [18], and cardiac surgery [19]. The simplicity and safety of the ESP block has been proposed as its main advantages. Potentially, a catheter placed at the ESP plane allows continuous infusion and prolonged analgesia [19].

Given the importance of providing adequate analgesia in VATS procedures and lack of consensus among surgeons and anesthesiologists for the optimal analgesic technique, we planned to conduct a randomized controlled trial (RCT) to compare continuous ESP blockade versus surgeon-performed ICNBs in patients receiving VATS lobectomy or wedge resections, with the primary objective of assessing the difference in opioid analgesia used. Before conducting the main trial, we conducted a pilot RCT, with the primary objective of establishing feasibility of patient recruitment and follow-up.

\section{Methods}

This was a two-arm randomized controlled feasibility trial conducted at St Joseph's Hospital, Hamilton, Ontario, Canada. The study was registered at clinicaltrials.gov with the registration number NCT03176667, and ethics approval was obtained from the Hamilton Integrated Research Ethics Board on July 18, 2017 (\#3012). Due to the unavailability of rupivacaine at the start of the study, as well as input regarding epinephrine from participating anesthesiologists, patients undergoing ESP were administered $30 \mathrm{ml}$ of $0.125 \%$ bupivacaine rather than $30 \mathrm{ml}$ of $0.5 \%$ ropivacaine with $5 \mu \mathrm{g} \mathrm{ml}^{-1}$ of epinephrine. Patient enrollment began December 2018.

\section{Participants}

Patients were screened for trial inclusion using the preoperative booking list. Adult patients ( $>18$ years) having elective unilateral VATS procedures with at least one overnight stay were included. Patients were excluded if they had one or more of the following: patient refusal, body mass index $>40 \mathrm{~kg} \mathrm{~m}^{-2}$, and contraindications to neuraxial analgesia techniques as per the American Society of Regional Anesthesia and Pain guidelines [20-22]; 
known allergy to local anesthetics; inability to use PCA due to language or comprehension barriers; procedures with a higher chance of open surgery; and patient on any regular opioids for $\geq 3$ months prior to surgery. During their pre-operative visit, a research assistant approached suitable patients, explained study procedures, and answered their questions. Patients had the opportunity to withdraw at any point. Baseline data, including demographics and history of comorbidities were collected at the same visit.

\section{Randomization, allocation, and blinding}

Patients were randomized to intervention (US-guided continuous ESP block) and control (surgeon-performed ICNB) groups with a 1:1 allocation ratio using a computerized randomization the day before surgery. Randomization was generated by a statistician not involved with the study, and the list was provided to the pharmacy. The group allocation was concealed and revealed to the anesthesiologist assigned to manage the surgical case the day before to ensure that a trained anesthesiologist was available to perform the preoperative ESP procedure and catheter placement. Due to the nature of the study interventions, patients and research personnel were not blinded.

\section{Intervention group}

In the intervention group, patients had a pre-operatively performed US-guided ESP block with catheter placement in a designated block room equipped with monitoring and resuscitation facilities. Intravenous (i.v.) access was established and standard American Society of Anesthesiologists monitors were applied. Sedation and anxiolytics were used as considered appropriate. Patients were placed in a sitting position and the area was sterilized with disposable swabs of $2 \%$ chlorhexidine in $70 \%$ isopropyl alcohol and then draped in a sterile fashion. A high-frequency linear US transducer (GE LOGIQe, Wauwatosa, WI, USA) was used in a longitudinal parasagittal orientation $3 \mathrm{~cm}$ lateral to the $\mathrm{T} 5$ spinous process. The trapezius, rhomboid major, and erector spinae muscles were identified superficial to the tip of the T5 transverse process. The patient's skin was anesthetized with 2\% lidocaine. A B-Braun Contiplex Echo 18 gauge Tuohy needle with the Contiplex Echo 20 gauge catheter (Braun Medical Inc., Bethlehem, PA, USA) was inserted using an in-plane superior-to-inferior approach to place the tip into the fascial plane on the deep (anterior) aspect of the erector spinae muscle. The location of the needle tip was confirmed by visible fluid spread lifting the erector spinae muscle off the bony shadow of the transverse process. A maximum of $30 \mathrm{ml}$ of $0.125 \%$ bupivacaine was injected in $5-\mathrm{ml}$ aliquots through the needle (maximum of $3 \mathrm{mg} \mathrm{kg}^{-1}$ ) followed by insertion of a 19 gauge catheter under direct vision $5 \mathrm{~cm}$ beyond the needle tip.

\section{Control group}

In the control group, the thoracic surgeons performed ICNBs from T4 to T11 on the operated side of the chest, at the end of surgical procedure. The ICNBs were performed using $0.25 \%$ bupivacaine with epinephrine and a volume of $5 \mathrm{ml}$ per block (maximum of $2.5 \mathrm{mg} \mathrm{kg}^{-1}$ ) was used.

\section{Anesthesia management}

The attending anesthesiologist provided a general anesthetic as per the routine institutional practice. For patients in the ESP block group, the attending anesthesiologist started a background infusion of bupivacaine $0.125 \%$ at $13 \mathrm{ml} \mathrm{h}^{-1}$ through the catheter after incision. At the end of the case a bolus of $0.125 \%$ bupivacaine $5 \mathrm{ml}$ was injected through the ESP catheter and the patient was extubated. Patients in the control group had ICNBs placed at the end of surgery, then extubated. PCA was initiated postoperatively in the recovery room.

\section{Postoperative management}

All patients were monitored in the post-anesthetic care unit (PACU) for stabilization as routine practice. If the patient complained of incisional pain of intensity of $>4$ / 10 on the numerical rating scale (NRS), the nurse provided boluses of hydromorphone $0.2-0.4 \mathrm{mg}$ i.v. every 7 min as needed to a maximum of $2 \mathrm{mg}$, after which PCA pumps were initiated. If the patient was allergic to hydromorphone, a morphine PCA was substituted. All patients were enrolled into the Acute Pain Service (APS) and were visited daily by a nurse practitioner and/or an anesthesiologist. In the ESP group, continuous ESP block was provided with a background infusion of $0.125 \%$ bupivacaine at $13 \mathrm{ml} \mathrm{h}^{-1}$.

The PCA pump was programmed to administer hydromorphone in boluses of 0.2 to $0.4 \mathrm{mg}$ i.v., with lockout time of 7 to $10 \mathrm{~min}$ to a maximum $6 \mathrm{mg}$ over 4 $\mathrm{h}$; or morphine in boluses of 1 to $2 \mathrm{mg}$ i.v., with a lockout time of 7 to $10 \mathrm{~min}$ to a maximum of $30 \mathrm{mg}$ over 4 h. Changes to PCA opioid doses was made by the APS team, as necessitated for each patient to target minimal or tolerable pain $(\mathrm{NRS}<4)$. In both groups, patients had multimodal analgesia, individualized based on the allergies, comorbidities, and patient tolerance: (1) acetaminophen $975 \mathrm{mg}$ orally/per rectum (PO/PR) for $48 \mathrm{~h}$ followed by $650 \mathrm{mg} \mathrm{PO} / \mathrm{PR}$ every $4 \mathrm{~h}$ as needed until discharge and (2) naproxen $500 \mathrm{mg}$ PO twice daily $\times$ eight doses (administer with food) or ketorolac $10 \mathrm{mg}$ i.v. four times daily $\times$ eight doses if patient is unable to ingest PO medications. PCA was discontinued upon 
removal of the chest tubes or when patient was ready for discharge, as is the current standard of practice at our hospital. ESP catheter infusion was discontinued $48 \mathrm{~h}$ after surgery or under any one or more of the following circumstances: chest drain removal, patient discharge, any signs of local infection or systemic infection, or any sign of mechanical dysfunction including leaking or inadvertent withdrawal of the catheter.

\section{Study outcomes}

\section{Primary outcomes (feasibility)}

To assess the feasibility of conducting a large RCT comparing the ESP versus ICNB, the following outcomes were evaluated: (1) recruitment rate (number of participants recruited per week and (2) proportion of inhospital follow-up until discharge. The study was considered feasible if the recruitment rate was greater than two patients per week and with complete $(100 \%)$ inhospital follow-up until discharge for the clinical outcomes.

\section{Secondary outcomes (clinical)}

Among the following clinical outcomes, differences in total opioid consumed over the first $24 \mathrm{~h}$ and $48 \mathrm{~h}$ were explored as primary outcomes for the larger RCT. We considered evaluating opioid use at both 24 and $48 \mathrm{~h}$ separately since a substantial percentage of patients were being discharged before $48 \mathrm{~h}$. For the main study, we plan to consider only one of the two as our primary outcome.

1. Differences in total opioid consumption over the first $24 \mathrm{~h}$ and $48 \mathrm{~h}$. This was collected using electronic medical records. Opioids will be considered as total hydromorphone equivalents for analysis.

2. Difference in pain scores "at rest" and "at movement" at the following time points: $1 \mathrm{~h}$ after entry at PACU, average pain score during the postoperative nights as collected during the morning APS rounds, and pain scores on each morning until discharge. Pain scores at movement were collected by asking patients to cough. Both pain scores were recorded using the patientreported NRS, an 11-point scale where 0 is no pain and 10 is the worst pain imaginable (Additional file 1: Appendix 1). The NRS is validated and considered easy to use [23].

3. Adverse effects: Difference in the incidence of postoperative nausea-vomiting, respiratory depression, itching, local anesthetic toxicity, catheter leakage and catheter migration, and infection around the catheter site. The definitions and scales of each of the outcomes are provided in Additional file 1: Appendix 1.

4. Presence of sensory blockade on the operated side of the chest was collected on the first postoperative morning. Sensory assessment was performed by trained data collectors for pin prick (using a blunt needle). The dermatome distribution of the blockade on the patient's anterior chest, midaxillary line, mid-clavicular line, and mid-scapular line was assessed using a blunt needle to test for loss of sensation to pinprick. Sensory testing was performed and recorded as shown in Additional file 1: Appendix 1.

5. Patient satisfaction with postoperative analgesia on the day of discharge. This was performed using a five-point Likert scale (Additional file 1: Appendix $1)$.

\section{Statistical analysis and sample size}

The analysis and reporting of the trial was performed in accordance with the Consolidated Standards of Reporting Trials (CONSORT) guidelines for pilot studies [24]. Baseline and intraoperative data were reported using means and standard deviations or median and interquartile range as appropriate. We analyzed as intent to treat (ITT) with each patient analyzed according to the group to which they were randomized. Clinical outcomes were compared using a two-sided test with a significance level of 0.05 . No subgroup tests were conducted. As suggested in literature for pilot studies, we considered a sample size of 12 patients per group to demonstrate feasibility $[24,25]$.

\section{Data collection and confidentiality}

Patients were noted using a study ID to keep their information anonymous. The patient's study ID and corresponding hospital medical record number were recorded in an Excel file in a password-encrypted USB that was securely stored in a locked cabinet. Data collection was done using paper forms and transferred to REDCap for secure storage and analysis. Data were collected by research assistants and the APS nurse practitioner (KD). The data collection forms were also kept in the locked cabinet.

\section{Results}

The study was carried out from November 2018 to February 2019 . Of the 54 patients meeting eligibility criteria 24 patients were consented and randomized to the intervention $(n=12)$ and control $(n=12)$ groups (Fig. 1). Table 1 and Table 2 list the baseline and intraoperative characteristics, respectively. The most common procedure performed was VATS lobectomy $(50 \%)$, followed by thoracoscopic wedge resections $(42 \%)$. Two cases $(8 \%$; 


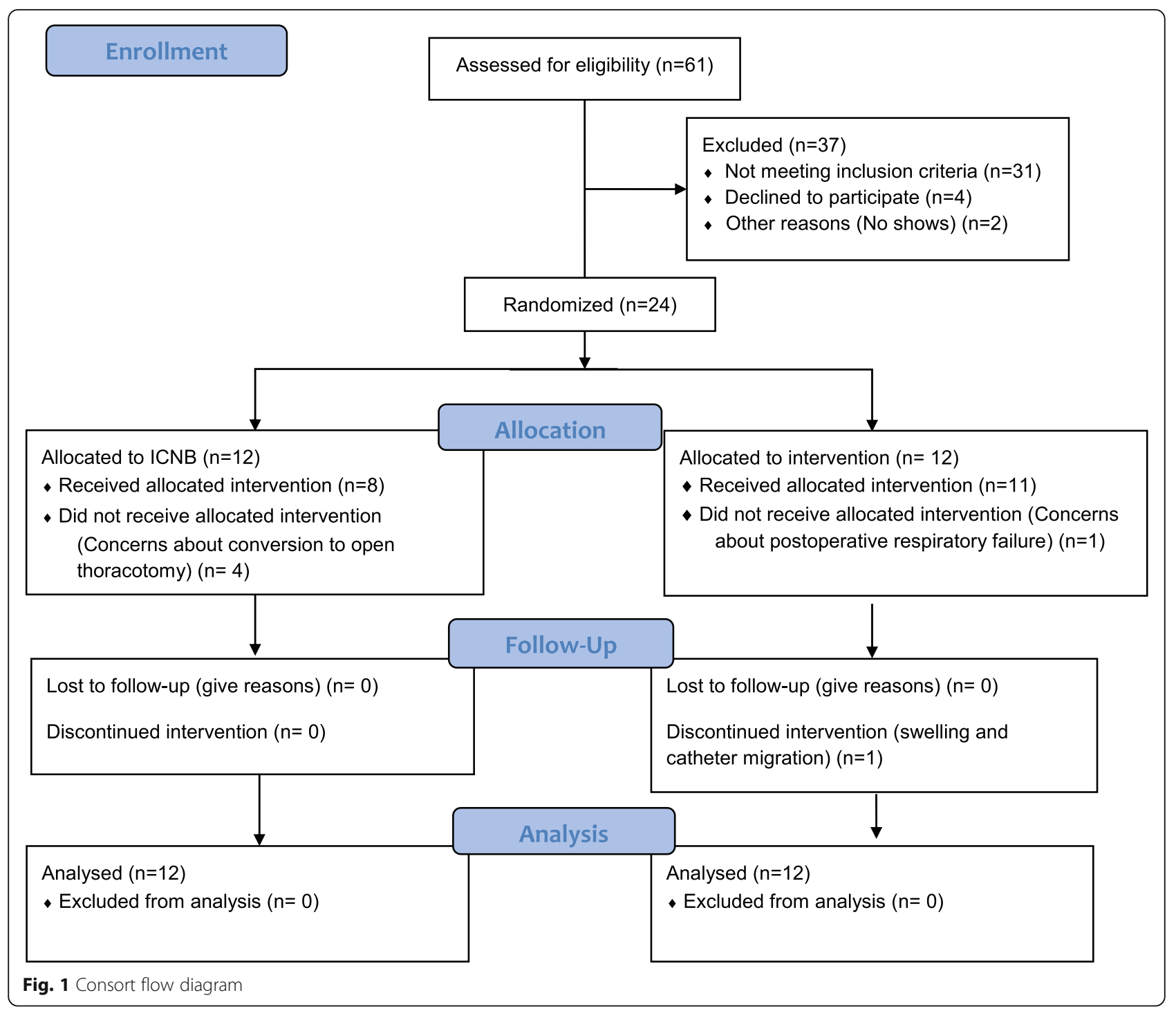

both from the intervention group) were converted to open lobectomy. The rate of adherence to the assigned treatment was $92 \%$ in the intervention group and $67 \%$ in the control group. Epidural catheters were inserted in five patients; four with a higher chance of converting to open surgeries intraoperatively and one patient about

Table 1 Baseline characteristics

\begin{tabular}{|c|c|c|}
\hline Baseline characteristics & Control $(n=12)$ & Intervention $(n=12)$ \\
\hline Age, years; mean $\pm S D$ & $66.50 \pm 8.44$ & $71.33 \pm 9.00$ \\
\hline Females; $n(\%)$ & $10(83.33)$ & $7(58.33)$ \\
\hline $\mathrm{BMI}, \mathrm{kg} \mathrm{m}^{-2}$; mean $\pm \mathrm{SD}$ & $25.10 \pm 5.16$ & $28.34 \pm 4.03$ \\
\hline Weight, kg; mean \pm SD & $66.37 \pm 20.14$ & $77.81 \pm 13.21$ \\
\hline ASA $3^{\mathrm{a}} ; n(\%)$ & $7(58.33)$ & $7(58.33)$ \\
\hline ASA $4^{\mathrm{a}} ; n(\%)$ & $5(41.67)$ & $5(41.67)$ \\
\hline
\end{tabular}

${ }^{a}$ There were no participants belonging to ASA categories of 1,2 , or 5 . ASA American Society of Anesthesiologists, $B M I$ body mass index whom the treating anesthesiologist had concerns about postoperative ventilatory failure based on predicted forced expiratory volume in $1 \mathrm{~s}$.

\section{Primary outcomes}

We were successful in demonstrating feasibility for both of our primary outcomes. As the recruitment happened over 58 days (11.6 weeks), the number of patients recruited per week was $>2$ and we successfully followed all patients for clinical outcomes during their hospital stay.

\section{Clinical outcomes}

Clinical outcomes are reported in Table 3 and Figs. 2, 3 , and 4 . As a feasibility trial, the trial was not designed to test for statistical significance within these outcomes. The mean opioid consumption was less in the intervention group over the first $24 \mathrm{~h}$; mean difference (MD) of 1.63 (95\% confidence interval [CI] 
Table 2 Intraoperative characteristics

\begin{tabular}{|c|c|c|}
\hline Intraoperative characteristics & Control $(n=12)$ & Intervention $(n=12)$ \\
\hline \multicolumn{3}{|l|}{ Diagnosis; $n(\%)$} \\
\hline Lung cancer & $8(66.7)$ & $9(75)$ \\
\hline Lung nodule & $4(33.3)$ & $3(25)$ \\
\hline \multicolumn{3}{|l|}{ Type of surgery; $n(\%)$} \\
\hline VATS lobectomy & $7(58)$ & $5(42)$ \\
\hline Thoracoscopic wedge resection & $5(42)$ & $5(42)$ \\
\hline Converted open lobectomy & 0 & $2(16)$ \\
\hline \multicolumn{3}{|l|}{ Type of analgesia; $n(\%)$} \\
\hline Epidural & $4(33.3)$ & $1(8)$ \\
\hline Adherence to assigned analgesia modality; $n(\%)$ & $8(66.7)^{a}$ & $11(91.7)^{\mathrm{a}}$ \\
\hline
\end{tabular}

andividuals who did not receive the correct assigned treatment (ESP or ICNB) received a thoracic epidural. ESP erector spinae plane block, ICNB intercostal nerve block

-1.20 to 4.45$)$, and $48 \mathrm{~h}, \mathrm{MD}$ of 2.34 (95\% CI -1.93 to 6.61) (Table 3, Fig. 4). Average pain on the first postoperative morning (which consisted of the first postoperative evening pain score and first postoperative morning pain score) was recorded in all patients and showed minimal difference between the groups at rest: $\mathrm{MD} 0.71(95 \% \mathrm{CI}-1.47$ to 2.88$)$; and with movement: MD -0.17 (95\% CI -2.49 to 2.16). Before the second postoperative morning 10 study, patients had their chest tubes and their continuous analgesia (PCA and ESP infusion) removed. Hence, we only had 14 patients reporting their pain scores on the second postoperative morning. The pain scores (which consisted of the second postoperative day evening pain score and second postoperative day morning pain score) were less in the intervention group at rest: $\mathrm{MD} 1.65$ (95\% CI -0.89 to 4.19$)$; and with movement: MD 1.77 (95\% CI -0.86 to 4.41 ).

Table 3 Summary of secondary outcomes

\begin{tabular}{|c|c|c|c|}
\hline Outcome & Control & Treatment & MD $(95 \% \mathrm{Cl})$ \\
\hline Cumulative opioid consumption (mg) at $24 \mathrm{~h}$, mean \pm SD & $\begin{array}{l}5.28 \pm 4.17 \\
n=12\end{array}$ & $\begin{array}{l}3.66 \pm 2.22 \\
n=12\end{array}$ & $1.63(-1.20$ to 4.45$)$ \\
\hline Cumulative opioid consumption (mg) between $24-48 \mathrm{~h}$, mean \pm SD & $\begin{array}{l}2.61 \pm 3.77 \\
n=12\end{array}$ & $\begin{array}{l}1.89 \pm 2.91 \\
n=12\end{array}$ & $0.72(-2.14$ to 3.57$)$ \\
\hline Cumulative opioid consumption (mg), at $48 \mathrm{~h}$, mean $\pm \mathrm{SD}$ & $7.89 \pm 5.58$ & $5.55 \pm 4.44$ & $2.34(-1.93$ to 6.61$)$ \\
\hline \multicolumn{4}{|l|}{ Pain score at rest ${ }^{\mathrm{a}}$} \\
\hline Average pain after $24 \mathrm{~h}$, mean \pm SD & $\begin{array}{l}3.75 \pm 2.18 \\
n=12\end{array}$ & $\begin{array}{l}3.04 \pm 2.90 \\
n=12\end{array}$ & $0.71(-1.47$ to 2.88$)$ \\
\hline Average pain after $48 \mathrm{hr}$, mean $\pm \mathrm{SD}$ & $\begin{array}{l}3.08 \pm 2.42 \\
n=6\end{array}$ & $\begin{array}{l}1.44 \pm 1.95 \\
n=8\end{array}$ & $1.65(-0.89$ to 4.19$)$ \\
\hline \multicolumn{4}{|l|}{ Pain scores with movement ${ }^{\mathrm{a}}$} \\
\hline Average pain after $24 \mathrm{~h}$; mean \pm SD & $\begin{array}{l}5.17 \pm 2.61 \\
n=12\end{array}$ & $\begin{array}{l}5.33 \pm 2.88 \\
n=12\end{array}$ & $-0.17(-2.49$ to 2.16$)$ \\
\hline Average pain after $48 \mathrm{~h}$, mean $\pm \mathrm{SD}$ & $\begin{array}{l}4.08 \pm 1.69 \\
n=6\end{array}$ & $\begin{array}{l}2.31 \pm 2.56 \\
n=8\end{array}$ & $1.77(-0.86$ to 4.41$)$ \\
\hline Adverse event: Catheter migration & 0 & 1 & \\
\hline Adverse event: Catheter disconnection & 0 & 1 & \\
\hline \multicolumn{4}{|l|}{ Patient satisfaction, $n(\%)$} \\
\hline Very satisfied & $7(58.33)$ & $8(66.67)$ & \\
\hline Satisfied & $3(25.00)$ & $3(25.00)$ & \\
\hline Neutral & $2(16.67)$ & $1(8.33)$ & \\
\hline Required treatment for nausea and vomiting in PACU, $n(\%)$ & $1(8.33)$ & $2(16.67)$ & . \\
\hline Required treatment for nausea and vomiting in the first $24 \mathrm{~h}$ postoperatively (excluding PACU), $n$ (\%) & $6(50)$ & $3(25)$ & . \\
\hline
\end{tabular}

${ }^{a}$ Numerical rating scale, an 11-point scale where 0 is no pain and 10 is the worst pain imaginable. MD mean difference, $P A C U$ post-anesthetic care unit 


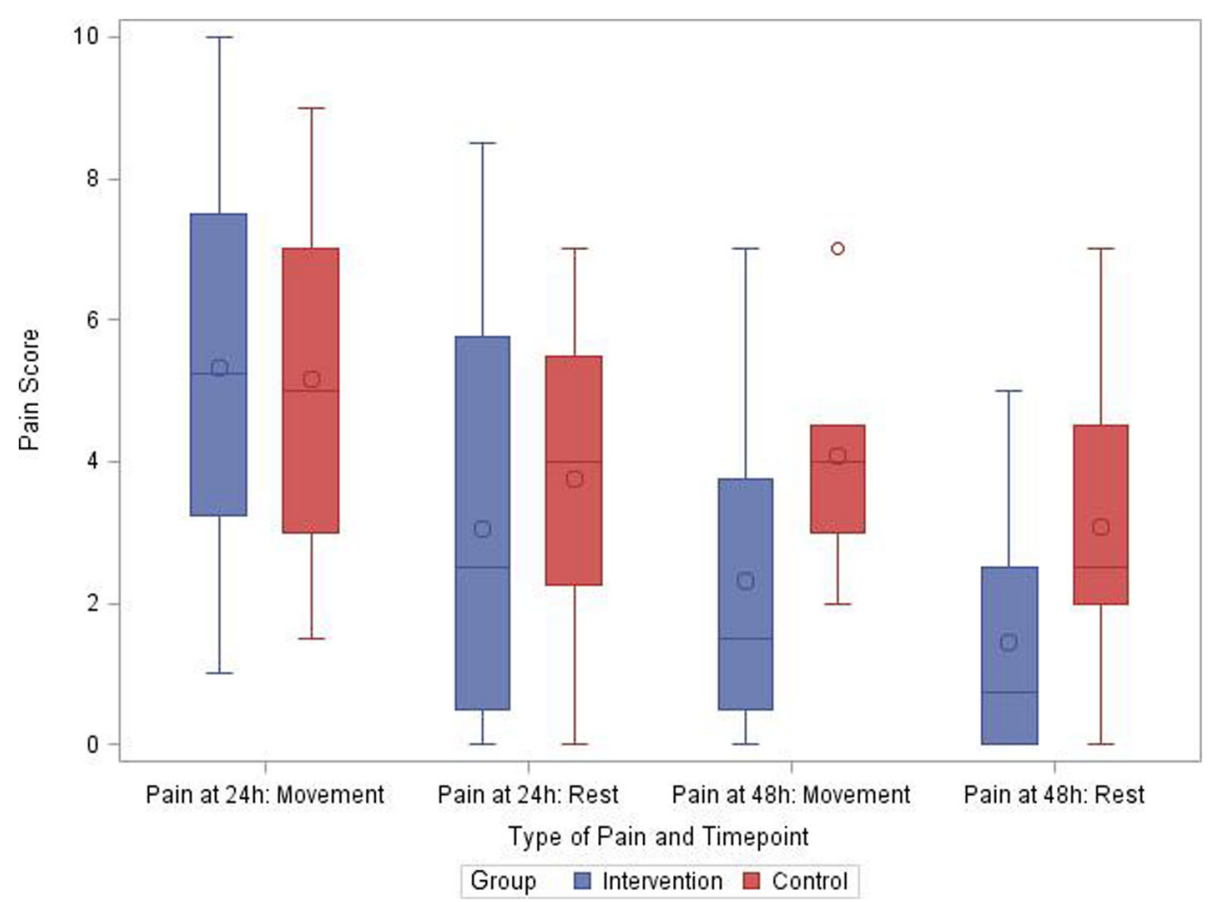

Fig. 2 Pain scores (box and whisker plot)

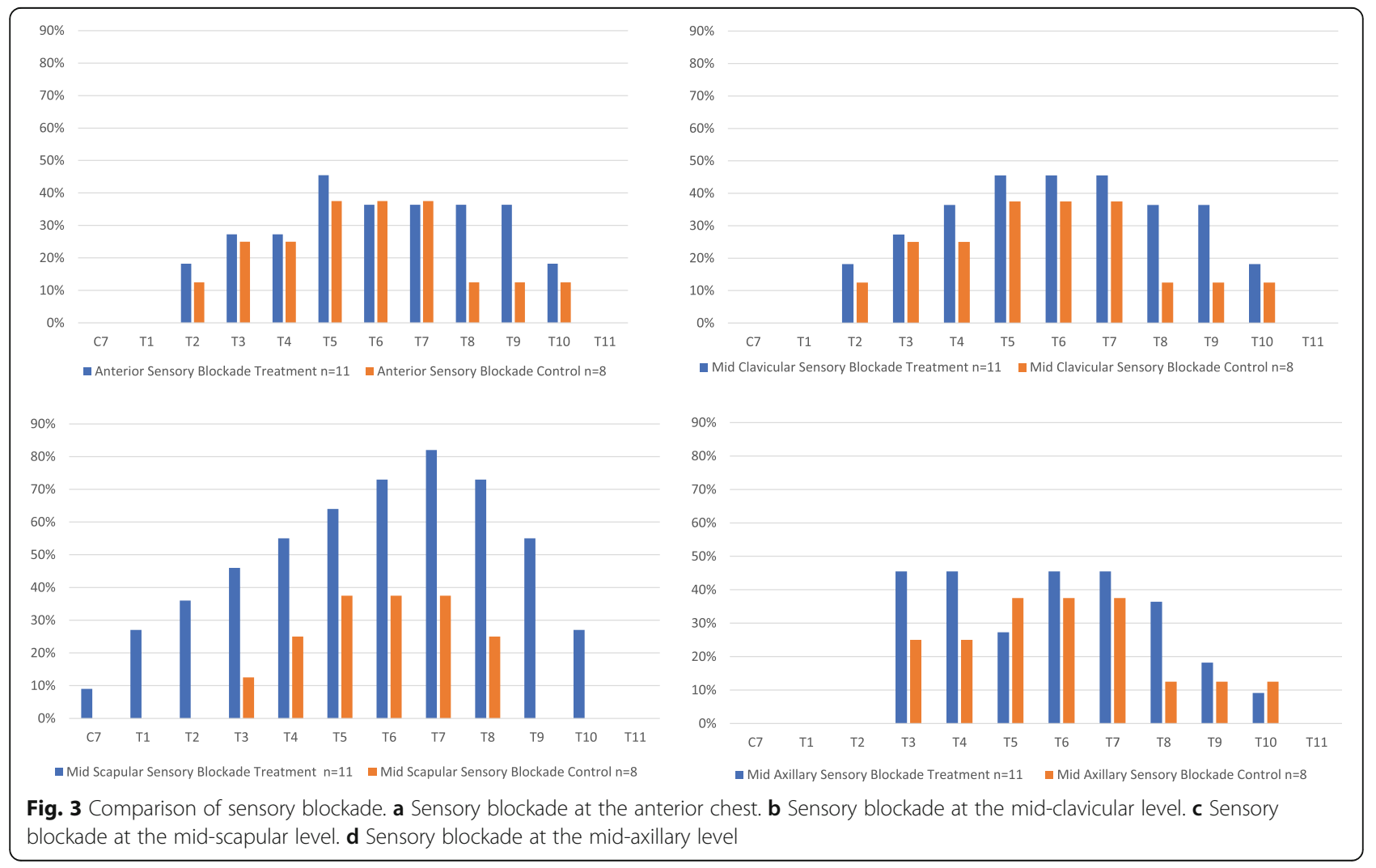




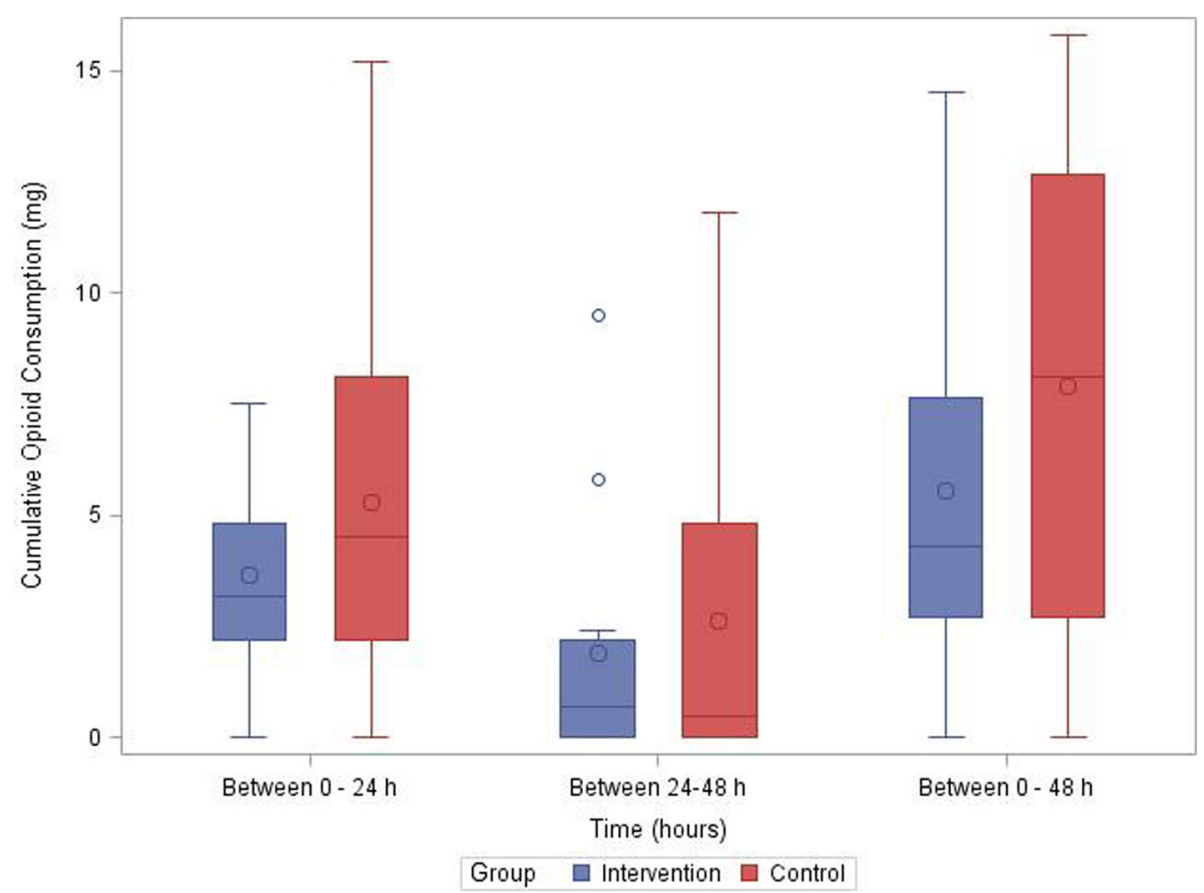

Fig. 4 Cumulative opioid consumption (box and whisker plot)

Two individuals (both in the intervention group) experienced at least one adverse event: one event was catheter migration at $24 \mathrm{~h}$, and the second was an accidental catheter disconnection. Nine individuals needed treatment for nausea and vomiting within $24 \mathrm{~h}$, in which three also required treatment in PACU. Among the five categories of patient satisfaction, there were no patients in either group that recorded dissatisfied or completely dissatisfied. In the control group, $58 \%$ were very satisfied, $25 \%$ were satisfied, and $17 \%$ were neutral. In the treatment group, $67 \%$ were very satisfied, $25 \%$ were satisfied, and $8 \%$ were neutral.

The presence of sensory blockade on the operative side of the thorax in each group is shown according to dermatome in Fig. 3. Figure 3a indicates that the difference between the two groups was more obvious at the mid-scapular line compared with other areas. Within the intervention group, peak blockade was observed at dermatomes T5, T6, and T7 for most areas and the percentage of patients who had such blockade were less than $50 \%$ of patients, except at the midscapular line.

\section{Discussion}

In this pilot trial, we were able to demonstrate the feasibility of conducting an RCT comparing continuous ESP analgesia versus ICNB in patients undergoing VATS. We were able to recruit more than two patients per week and follow all patients during their hospital stay. We also observed that the study intervention had a positive influence on the opioid consumption and postoperative pain scores. As a feasibility trial, it was not powered to assess for clinical outcomes. There were no serious adverse clinical events that posed a threat to patient safety or resulted in any known morbidity or mortality. Based on our pilot study, and using the same comparators, we would need a total of 63 patients/group for a difference of $1.63 \mathrm{mg}$ between the groups. We provide estimates of sample size with other variations to mean difference and SDs of the treatment and comparator group in our supplemental table (Additional file 2: Appendix 2).

A few other trials have explored the effectiveness of single-shot ESP blocks in VATS and thoracotomies. However, not many studies have assessed the value of continuous ESP block for VATS patients. Fang et al. [26] demonstrated that, compared with a single PVB, a single-shot ESP block with $20 \mathrm{ml}$ of $0.25 \%$ bupivacaine provided no differences in pain scores and postoperative opioid consumption in patients undergoing thoracotomies, with less incidence of hypotension. Chen et al. [27] compared ICNB, a single-shot ESP block, and multiple paravertebral nerve blocks for VATS. They found that PVBs provided superior analgesia with reduction in visual analog scale scores and opioid consumption in comparison to both single-shot ESP blocks and intercostal blocks. However, when single-shot ESP blocks and 
intercostal blocks were compared with one another they were equally effective in reducing pain. Gaballah et al. [28] reported that patients treated with a single ESP block had lower visual analogue scale scores and longer time to first analgesia compared with patients who received a single shot of serratus plane block. The results of our study suggest similar findings to these studies, showing that a continuous ESP block may provide superior analgesia through decreased pain scores and decreased opioid consumption compared with a singleshot intercostal block. A recent trial observed that analgesia provided by ESP was non-inferior to PVB for VATS resections, and ESP also resulted in lower plasma concentrations of levobupivacaine [29]. This further substantiates the relative safety of ESP over PVB.

One important observation that warrants attention and planning prior to a larger scale RCT using our study design is how to optimize adherence to the assigned interventions. As noted above, five of the 24 patients received a thoracic epidural and four in the control group and one in the intervention group. In four patients, epidurals were inserted in consideration of a higher chance of converting to open surgeries intraoperatively, but only two patients were actually converted to thoracotomy. Our trial was not blinded to patients and healthcare providers and there may be a bias toward considering an epidural in the control group for patients with a higher chance of open conversion. Because continuous epidural is a potent intervention for pain relief and reducing opioids, it has the potential to influence clinical outcomes for the group. It is important to find ways to improve adherence as it may increase the chances of type II error (false negative). Possible solutions include reminding physicians that all procedures have a risk of being converted to open thoracotomy, and routinely placed thoracic epidurals are not currently the standard of practice at our institution. Furthermore, thoracic epidurals may also be placed postoperatively if indicated. Tightening the selection criteria so that only suitable patients are randomized can be considered. However, tightening the selection criteria has important implications to study recruitment. There have been suggestions that ITT analysis may not always be appropriate in circumstances such as the above where inappropriate patients were included, and it might be better to go with the recommendations of an adjudication committee for each case inclusion. Finally, another point that should be mentioned is the option to exclude any case that gets converted to open thoracotomy.

It has been observed in the literature that the ESP block has variable dermatomal spread. We note that in comparison to ICNB from T4-T8 placed by the surgeon, an ESP placed at T5 covered more dermatomes in our study. Other reports have shown that ESP has the potential for a wider spread. It is probably likely that more obvious blockade at the mid scapular line is explained by predominant blockade of posterior branches. Using computed tomography with contrast, Forero et al. [17] demonstrated that injection of $20 \mathrm{ml}$ of $0.5 \%$ bupivacaine at $\mathrm{T} 5$ showed spread between $\mathrm{T} 1$ and $\mathrm{T} 11$ vertebra. Scimia et al. [30] similarly showed that a continuous ESP block at T6 in a patient receiving VATS lobectomy had T2T10 dermatomal spread using a cold test in the postoperative period.

We believe our primary clinical outcome of reduction in opioid usage is more appropriate than pain scores. Since opioid usage was based on PCA needs, it still reflects pain perceived by a patient indirectly if one assumes that opioid needs are based on individual pain tolerance. Opioid usage was observed to be lower in the intervention arm at all points. The decrease in opioid consumption may also explain the decrease in anti-emetic drug administration seen in the ESP and PCA arm. Although we did not intend to limit the inclusion based on the ASA score, all of our patients belonged to either ASA 3 or 4 risk category. We recognize that this might potentially affect the external validity of the study results and hence we plan to ensure inclusion of a wider range of ASA score patients for our main trial.

There are a few important limitations of our study. This pilot RCT was not blinded; hence, it carries a higher potential for observation bias. It should also be emphasized that this is a pilot study and it was not designed or powered to provide clinical inference. As mentioned above, there were five patients that had an epidural, indicating non-compliance with study protocol. In view of this, we could consider patient selection criteria as a limitation that needs to be improved in our main trial.

\section{Conclusions}

In conclusion, we conducted a pilot, non-blinded RCT comparing continuous ESP blockade with surgeon-performed ICNB in patients having VATS resections. Our trial demonstrated that it would be feasible with modifications to conduct a fully powered RCT. We further demonstrate that this type of trial is safe and that there are indications of lower opioid consumption and superior analgesia in the ESP arm. However, more research is required to determine whether this effect is clinically meaningful and statistically significant. 


\section{Supplementary Information}

The online version contains supplementary material available at https://doi. org/10.1186/s40814-021-00801-7.

Additional file 1: Appendix 1 A supplementary document that contains definitions and scales of each outcome, and diagrams of sensory testing.

Additional file 2: Appendix 2 Appendix_Calculation of group sample size. A supplementary document that presents the data for sample size estimates for the main trial (primary outcome, cumulative opioid consumption at $24 \mathrm{~h}$ ).

\section{Abbreviations}

APS: Acute Pain Service; ASA: American Society of Anesthesiologists; BMI: Body mass index; Cl: Confidence interval; CONSORT: Consolidated Standards of Reporting Trials; ESP: Erector spinae plane block; ICNB: Intercostal nerve blockade; ITT: Intent to treat; i.v: Intravenous; MD: Mean difference; PCA: Patient-controlled analgesia; PO: Orally; PR: Per rectum; PVB: Paravertebral block; RCT: Randomized controlled trial; TEA: Thoracic epidural analgesia; US: Ultrasound; VATS: Video-assisted thoracoscopic surgery

\section{Acknowledgements}

None declared.

\section{Previous presentation}

None declared.

\section{Authors' contributions}

DH: Study conception, data acquisition and interpretation, drafting and revising the manuscript, and final approval of the version to be published. WS: Study conception, data acquisition and interpretation, drafting and revising the manuscript, and final approval of the version to be published. PM: Study conception, performance of interventional procedures, drafting and revising the manuscript, and final approval of the version to be published. TO: Performance of interventional procedures, data acquisition and interpretation, drafting and revising the manuscript, and final approval of the version to be published. YS: Data acquisition and interpretation, drafting and revising the manuscript, and final approval of the version to be published. CF: Data acquisition and interpretation, drafting and revising the manuscript, and final approval of the version to be published. WH: Data acquisition and interpretation, drafting and revising the manuscript, and final approval of the version to be published. JA: Data acquisition and interpretation, drafting and revising the manuscript, and final approval of the version to be published. MF: Data acquisition and interpretation, drafting and revising the manuscript, and final approval of the version to be published. KD: Data acquisition and interpretation, drafting and revising the manuscript, and final approval of the version to be published. TV: Data analysis, drafting and revising the manuscript, and final approval of the version to be published. LT: Study conception, data acquisition and interpretation, drafting and revising the manuscript, and final approval of the version to be published. HS: Study conception and supervision, data acquisition and interpretation, drafting and revising the manuscript, and final approval of the version to be published. The authors read and approved the final manuscript.

\section{Funding}

We received funding from the Regional Medical Association at McMaster in the form of a post graduate award in 2018. The funder had no role in study design; data collection, analysis, and interpretation; or writing the manuscript. HS is supported by Canadian Anesthesia Research Foundation as a Career Scientist Award 2018-2020.

\section{Availability of data and materials}

Data are available from the corresponding author upon request.

\section{Ethics approval and consent to participate}

Ethics approval was obtained from the Hamilton Integrated Research Ethics Board on July 18, 2017 (\#3012). Informed consent was obtained from each patient participant.

\section{Consent for publication}

N/A.

\section{Competing interests}

The authors declare that they have no competing interests.

\section{Author details}

${ }^{1}$ Department of Anesthesia, McMaster University, Hamilton, Ontario, Canada. ${ }^{2}$ Department of Surgery, McMaster University, Hamilton, Ontario, Canada. ${ }^{3}$ Department of Anesthesia, St. Joseph's Health Care Hamilton, Hamilton, Ontario, Canada. ${ }^{4}$ Health Research Methods, Evidence and Impact, McMaster University, Hamilton, Ontario, Canada.

Received: 18 August 2020 Accepted: 16 February 2021

Published online: 24 February 2021

\section{References}

1. Kaseda S, Aoki T, Hangai N, Shimizu K. Better pulmonary function and prognosis with video-assisted thoracic surgery than with thoracotomy. Ann Thorac Surg. 2000;70:1644-6.

2. McKenna RJ, Houck W, Fuller CB. Video-assisted thoracic surgery lobectomy: experience with 1,100 cases. Ann Thorac Surg. 2006;81:421-6.

3. Demmy TL, Curtis JJ. Minimally invasive lobectomy directed toward frail and high-risk patients: a case-control study. Ann Thorac Surg. 1999;68:194-200.

4. Scott WJ, Allen MS, Darling G, Meyers B, Decker PA, Putnam JB, et al. Videoassisted thoracic surgery versus open lobectomy for lung cancer: a secondary analysis of data from the American College of Surgeons Oncology Group Z0030 randomized clinical trial. J Thorac Cardiovasc Surg. 2010;139:976-83.

5. Katz J, Jackson M, Kavanagh BP, Sandler AN. Acute pain after thoracic surgery predicts long-term post-thoracotomy pain. Clin J Pain. 1996;12:50-5.

6. Zhang S, Huang Q, Xie J, Xu B, Cao G, Pei F. Factors influencing postoperative length of stay in an enhanced recovery after surgery program for primary total knee arthroplasty. J Orthop Surg Res. 2018;13:29.

7. Jamison RN, Ross MJ, Hoopman P, Griffin F, Levy J, Daly M, et al. Assessment of postoperative pain management: patient satisfaction and perceived helpfulness. Clin J Pain. 1997:13:229-36.

8. Joshi GP, Bonnet F, Shah R, Wilkinson RC, Camu F, Fischer B, et al. A systematic review of randomized trials evaluating regional techniques for postthoracotomy analgesia. Anesth Analg. 2008;107:1026-40.

9. Kim JA, Kim TH, Yang M, Gwak MS, Kim GS, Kim MJ, et al. Is intravenous patient controlled analgesia enough for pain control in patients who underwent thoracoscopy? J Korean Med Sci. 2009;24:930-5.

10. Fernandez MI, Martin-Ucar AE, Lee HD, West KJ, Wyatt R, Waller DA. Does a thoracic epidural confer any additional benefit following video-assisted thoracoscopic pleurectomy for primary spontaneous pneumothorax? Eur J Cardiothoracic Surg. 2005;27:671-4.

11. Yoshioka M, Mori T, Kobayashi H, Iwatani K, Yoshimoto K, Terasaki H, et al. The efficacy of epidural analgesia after video-assisted thoracoscopic surgery: a randomized control study. Ann Thorac Cardiovasc Surg. 2006;12:313-8.

12. Karmakar MK, Chung DC. Variability of a thoracic paravertebral block. Are we ignoring the endothoracic fascia? Reg Anesth Pain Med. 2000;25:325-7.

13. Huan S, Deng Y, Wang J, Ji Y, Yin G. Efficacy and safety of paravertebral block versus intercostal nerve block in thoracic surgery and breast surgery: a systematic review and meta-analysis. PloS one. 2020;15(10):e0237363.

14. Wurnig PN, Lackner $\mathrm{H}$, Teiner $\mathrm{C}$, et al. Is intercostal block for pain management in thoracic surgery more successful than epidural anaesthesia? Eur J Cardio-Thorac Surg. 2002;21(6):1115-9.

15. Bolotin G, Lazarovici H, Uretzky G, Zlotnick AY, Tamir A, Saute M. The efficacy of intraoperative internal intercostal nerve block during videoassisted thoracic surgery on postoperative pain. Ann Thorac Surg. 2000;70: 1872-5.

16. Taylor R, Massey S, Stuart-Smith K. Postoperative analgesia in video-assisted thoracoscopy: the role of intercostal blockade. J Cardiothorac Vasc Anesth. 2004; 18:317-21.

17. Forero M, Adhikary SD, Lopez H, Tsui C, Chin KJ. The erector spinae plane block a novel analgesic technique in thoracic neuropathic pain. Reg Anesth Pain Med. 2016;41:621-7.

18. Bonvicini D, Boscolo-Berto R, De Cassai A, et al. Anatomical basis of erector spinae plane block: a dissection and histotopographic pilot study. J Anesth. 2021;35:102-11. 
19. Forero M, Rajarathinam M, Adhikary S, Chin KJ. Continuous erector spinae plane block for rescue analgesia in thoracotomy after epidural failure: a case report. A A Case Rep. 2017;8:254-6.

20. American Society of Anesthesiologists Task Force on infectious complications associated with neuraxial techniques. Practice advisory for the prevention, diagnosis, and management of infectious complications associated with neuraxial techniques: a report by the American Society of Anesthesiologists Task Force on infectious complications associated with neuraxial techniques. Anesthesiology. 2010;112:530-45.

21. Horlocker $T$, Vandermeuelen E, Kopp SL, Gogarten W, Leffert LR, Benzon $H T$. Regional anesthesia in the patient receiving antithrombotic or thrombolytic therapy: American Society of Regional Anesthesia and Pain Medicine evidence-based guidelines (fourth edition). Reg Anesth Pain Med. 2018;43:263-309

22. Neal JM, Barrington MJ, Brull R, Hadzic A, Hebl JR, Horlocker TT, et al. The second ASRA practice advisory on neurologic complications associated with regional anesthesia and pain medicine: executive summary 2015. Reg Anesth Pain Med. 2015;40:401-30.

23. Bijur PE, Latimer $C T$, Gallagher EJ. Validation of a verbally administered numerical rating scale of acute pain for use in the emergency department. Acad Emerg Med. 2003;10:390-2.

24. Eldridge SM, Chan CL, Campbell MJ, Bond CM, Hopewell S, Thabane L, et al. CONSORT 2010 statement: extension to randomised pilot and feasibility trials. Pilot Feasibility Stud. 2016;2:64.

25. Julious SA. Sample size of 12 per group rule of thumb for a pilot study. Pharmaceut Statist. 2005:4:287-91.

26. Fang B, Wang Z, Huang X. Ultrasound-guided preoperative single-dose erector spinae plane block provides comparable analgesia to thoracic paravertebral block following thoracotomy: a single center randomized controlled double-blind study. Ann Transl Med. 2019;7:174.

27. Chen N, Qiao Q, Chen R, Xu Q, Zhang Y, Tian Y. The effect of ultrasoundguided intercostal nerve block, single-injection erector spinae plane block and multiple-injection paravertebral block on postoperative analgesia in thoracoscopic surgery: a randomized, double-blinded, clinical trial. J Clin Anesth. 2020:59:106-11.

28. Gaballah KM, Soltan WA, Bahgat NM. Ultrasound-guided serratus plane block versus erector spinae block for postoperative analgesia after videoassisted thoracoscopy: a pilot randomized controlled trial. J Cardiothorac Vasc Anesth. 2019:33:1946-53.

29. Taketa Y, Irisawa Y, Fujitani T. Comparison of ultrasound-guided erector spinae plane block and thoracic paravertebral block for postoperative analgesia after video-assisted thoracic surgery: a prospective randomized non-inferiority trial. Reg Anesth Pain Med. 2019;rapm-2019-100827. Online ahead of print.

30. Scimia P, Ricci EB, Droghetti A, Fusco P. The ultrasound-guided continuous erector spinae plane block for postoperative analgesia in video-assisted thoracoscopic lobectomy. Reg Anesth Pain Med. 2017;42:538-9.

\section{Publisher's Note}

Springer Nature remains neutral with regard to jurisdictional claims in published maps and institutional affiliations.

Ready to submit your research? Choose BMC and benefit from:

- fast, convenient online submission

- thorough peer review by experienced researchers in your field

- rapid publication on acceptance

- support for research data, including large and complex data types

- gold Open Access which fosters wider collaboration and increased citations

- maximum visibility for your research: over $100 \mathrm{M}$ website views per year

At BMC, research is always in progress.

Learn more biomedcentral.com/submissions 